\title{
How do starspots influence the transit timing variations of exoplanets? Simulations of individual and consecutive transits
}

\author{
P. Ioannidis, K. F. Huber, and J. H. M. M. Schmitt
}

\author{
Hamburger Sternwarte, Universität Hamburg, Gojenbergsweg 112, 21029 Hamburg, Germany \\ e-mail: pioannidis@hs.uni-hamburg.de
}

Received 13 August 2015 / Accepted 9 October 2015

\section{ABSTRACT}

\begin{abstract}
Transit timing variations (TTVs) of exoplanets are normally interpreted as the consequence of gravitational interaction with additional bodies in the system. However, TTVs can also be caused by deformations of the system transits by starspots, which might thus pose a serious complication in their interpretation. We therefore simulate transit light curves deformed by spot-crossing events for different properties of the stellar surface and the planet, such as starspot position, limb darkening, planetary period, and impact parameter. Mid-transit times determined from these simulations can be significantly shifted with respect to the input values; these shifts cannot be larger than $1 \%$ of the transit duration and depend very strongly on the longitudinal position of the spot during the transit and the transit duration. Consequently, TTVs with amplitudes larger than the above limit are very unlikely to be caused by starspots. We also investigate whether TTVs from sequences of consecutive transits with spot-crossing anomalies can be misinterpreted as the result of an additional body in the system. We use the Generalized Lomb-Scargle periodogram to search for periods in TTVs and conclude that low-amplitude TTVs with statistically significant periods around active stars are the most problematic cases. In those cases where the photometric precision is high enough to inspect the transit shapes for deformations it should be possible to identify TTVs caused by starspots; however, especially for cases with low signal-to-noise in transit (TSNR $\lesssim 15$ ) light curves it becomes quite difficult to reliably decide whether these periods come from starspots, physical companions in the system, or if they are random noise artifacts.
\end{abstract}

Key words. starspots - stars: activity - planetary systems - methods: numerical - eclipses - occultations

\section{Introduction}

The transits of a planet in front of its host star contain a wealth of fundamental information both on the planet and its host star. Using mid-transit times and studying their variations it is possible to measure the gravitational interaction of additional bodies that orbit the same center of mass. These transit timing variations (TTVs) can be used to determine the masses of planets in a multi-planet system and can even be used to infer the existence of additional (even low-mass) companions as has been shown, for example, by Agol et al. (2005). Although masses and additional companions can also be inferred from precise radial velocity (RV) measurements, the importance of the TTV method increases for planets around faint stars where accurate RV measurements are difficult to obtain.

Depending on the actual system configuration, TTVs can reach differences with respect to the linear period ephemeris of up to a few hours, especially for systems close to low-order mean motion resonances (Nesvorný et al. 2013). Although the determination of TTVs is possible from ground-based observations, the high precision of the data from the CoRoT (Baglin et al. 2006) and Kepler (Koch et al. 2010) space missions, along with the possibility of uninterrupted sequences of transit observations, is better suited for this task and has provided evidence of statistically significant TTVs in about 60 cases $^{1}$.

In addition, space-based photometry has shown that the light curve of a star can also be substantially disturbed by the effects of stellar activity. In particular, the transit profiles of planets with active host stars can be heavily distorted by dark spots

\footnotetext{
According to the list provided by http://exoplanet.org as of May 2015.
}

as observed, for example, in Tres-1 b (Rabus et al. 2009) or CoRoT-2 b (e.g., Wolter et al. 2009; Huber et al. 2009). This effect can be severe enough to introduce large systematic errors in the determination of the physical parameters of the planets, which can greatly exceed the formal statistical errors (Czesla et al. 2009; Oshagh et al. 2013).

Recently, there have been reports of the detection of lowamplitude TTVs, which are assumed to be caused by starspot signatures in the transit profiles (Sanchis-Ojeda et al. 2011; Fabrycky et al. 2012; Szabó et al. 2013; Mazeh et al. 2013). A first approach to studying the effect of starspots on TTVs was therefore made by Barros et al. (2013) to explain the $\sim 3$ min TTVs of the planet WASP-10 b, while a more general approach, including a variety of configurations between the starspot and the planet, was presented by Oshagh et al. (2013).

In this paper we simulate light curves of exoplanetary transits including spot-crossing events for a wider variety of the properties of the underlying star, starspot, and planet, and investigate the influence of these starspot signatures on the determination of mid-transit times. Furthermore, we study sequences of consecutive transits affected by starspots and evaluate the possibility of detecting a statistically significant signal in the TTVs that could mimic the TTV signal of a physical body.

The structure of our paper is as follows. In Sect. 2 we describe our approach for generating light curves in general, including our basic assumptions on the properties of the stellar surface and the planet. In Sect. 3 we state the specific questions we want to investigate and describe the individual simulation setups in detail. In Sect. 4 we present the results for each of our previously described simulation setups. In Sect. 5 we explore the possibility for the spot-crossing events to create spurious TTVs in transit sequences. In Sect. 6 we present a comparison of our 
results with real TTV observations. Finally, in Sect. 7 we discuss our results, and conclude with a summary in Sect. 8.

\section{Light curve simulations}

\subsection{Light curves of stars with spots}

A rotating star with a persistent dark spot on its surface produces a periodic modulation of its light curve. However, the exact shape of the photometric variation depends on the properties of the star and of the spot. If the life time $\tau_{\mathrm{sp}}$ of the spots (or rather active regions) is much longer than the stellar rotation pe$\operatorname{riod} P_{\star}$, the observed flux variations will be similar from one rotation to the next.

In many of the CoRoT and Kepler light curves of active stars with $\tau_{\mathrm{sp}} / P_{\star}>1$, the observed photometric variations consist of one or two pronounced dips per rotation, usually without the presence of any intervals where the stellar flux stays constant. This suggests that there are always at least a few spots located on the star that rotate on and off the visible hemisphere. The peak-to-peak amplitude of such photometric variations can be as high as several percent of the total flux (Basri et al. 2013).

Throughout our simulations we make the simplified assumption that the stellar surface is covered by only one large spot with a temperature lower than the photospheric temperature. We also assume that the spot properties do not change, i.e., we do not consider spot evolution effects or any internal spot structure such as penumbra and umbra. We adopt a center-to-limb intensity variation of the stellar photosphere according to the specific temperature, which is parameterized using a quadratic limb-darkening law.

\subsection{Spot-crossing events}

In the following we briefly explain our mathematical description of the photometric effects we expect during a spot-crossing event. During a planetary transit the total flux $F_{0}$ of the star decreases to $F_{\text {tr }}$ because the planet covers part of the stellar disk. We obtain for the ratio $F_{\mathrm{tr}} / F_{0}$ the expression

$\frac{F_{\mathrm{tr}}}{F_{0}}=1-\frac{R_{\mathrm{pl}}^{2}}{R_{\star}^{2}}=1-f_{\mathrm{pl}}$,

where $R_{\mathrm{pl}}$ denotes the planetary radius and $R_{\star}$ the stellar radius. We call $f_{\mathrm{pl}}$ the planetary filling factor. For the sake of simplicity, we assume that the planetary disk is completely dark; we ignore the limb-darkening (LD) effects and the ingress and egress phases when the stellar disk is only partially covered by the planet; however, the formalism is easily extended to cover these effects.

We now consider a spotted star with a spot filling factor $f_{\mathrm{sp}}$, which denotes the relative area covered by spots. We also introduce the relative spot temperature $t_{\mathrm{sp}}=T_{\mathrm{sp}} / T_{\star}$, where $T_{\mathrm{sp}}$ and $T_{\star}$ denote the temperatures of the spot and the photosphere, respectively. In this case the observed normalized flux of the star is not $F_{0}$, i.e., the flux seen from an undisturbed photosphere; rather, we see the "spotted" flux $F_{\text {sp }}$ given by the expression

$$
\frac{F_{\mathrm{sp}}}{F_{0}}=1-f_{\mathrm{sp}}\left(1-t_{\mathrm{sp}}^{4}\right)
$$

Now we consider the case of a spot-crossing event during a planetary transit and assume that the transiting planet is occulting a starspot either partially or totally. We let $d f_{\mathrm{pl}}$ denote the fraction of the planetary disk covering a spotted surface area, while

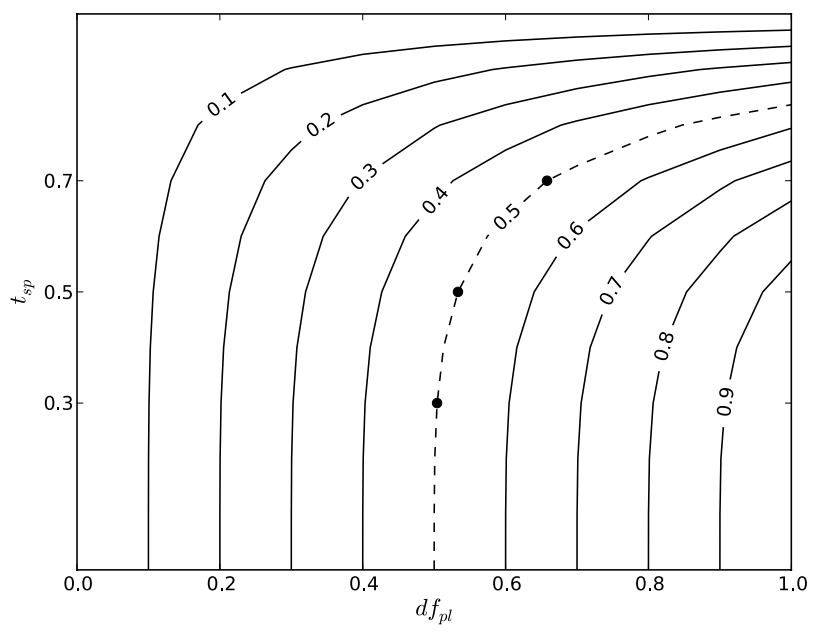

Fig. 1. Spot-crossing anomaly height $H$ (contours) as a function of the fraction of the planetary disk, which covers spotted surface area $d f_{\mathrm{pl}}$ and the spot temperature $t_{\mathrm{sp}}$. For our simulations we use values of $d f_{\mathrm{pl}}$ that correspond to spot temperatures equal to $0.3,0.5$, and 0.7 in order to reach $H=0.5$ (black dots along the dashed line).

$\left(1-d f_{\mathrm{pl}}\right)$ denotes the fraction of the planetary disk covering unspotted photosphere. In this case, the observed flux during the transit $F_{t r, s p}$ is given by the spotted flux $F_{\text {sp }}$, diminished by the relative flux of spotted and unspotted photosphere covered by the planet, i.e.,

$\frac{F_{\mathrm{tr}, \mathrm{sp}}}{F_{0}}=1-f_{\mathrm{sp}}\left(1-t_{\mathrm{sp}}^{4}\right)-f_{\mathrm{pl}}\left[1-d f_{\mathrm{pl}}\left(1-t_{\mathrm{sp}}^{4}\right)\right]$.

We usually work with transits normalized to the out-of-transit flux, which means we divide this equation by the contribution of the spot (Eq. (2)). This normalized flux $d$ is then defined by

$d=\frac{F_{\mathrm{tr}, \mathrm{sp}}}{F_{\mathrm{sp}}}=1-\frac{f_{\mathrm{pl}}\left[1-d f_{\mathrm{pl}}\left(1-t_{\mathrm{sp}}^{4}\right)\right]}{1-f_{\mathrm{sp}}\left(1-t_{\mathrm{sp}}^{4}\right)}$.

If the starspot only insignificantly contributes flux with respect to the unspotted surface $\left(t_{\mathrm{sp}} \approx 0\right)$, and if that spot is large enough that the planet covers only spotted surface regions $\left(d f_{\mathrm{pl}}=1\right)$, Eq. (4) becomes unity, i.e., the spot-crossing event would increase the flux to the out-of-transit level. We are not aware of any observations presented so far where this has been seen and we propose several reasons for this. First, although starspots are likely to be much cooler than photospheric temperatures, they still contribute a significant amount of flux. Second, most individual starspots are probably not as large as a planet. When we talk about starspots, we usually do not think of one large uniformly dark spot but of a densely spotted region made up of umbra, penumbra, and photospheric parts. However, such an active region might easily be much larger than the occulting planet, although the very dark (umbral) parts of the region are not. Third, a planet crossing over such a spotted area does not always have to cover the darkest parts of the spot but can cross near the periphery as well.

We introduce the height $H$ of the spot-crossing anomaly in a transit, which is given by the comparison between the relative flux $d$ in the cases with and without $\left(d_{d f_{\mathrm{pl}}=0}\right)$ a spot-crossing event.

$H=\frac{d-d_{d f_{\mathrm{pl}}=0}}{1-d_{d f_{\mathrm{pl}}=0}}=d f_{\mathrm{pl}}\left(1-t_{\mathrm{sp}}^{4}\right)$.

Figure 1 illustrates the dependence of $H$ on $d f_{\mathrm{pl}}$ and $t_{\mathrm{sp}}$; for $H=0$ there is no spot-crossing anomaly, while $H=1$ means 
that the increase is equal to the transit depth and, thus, the flux returns to the out-of-transit level. As discussed before, values of $H>0.5$ have not been observed so far and seem to be unlikely; therefore, we select those combinations of $d f_{\mathrm{pl}}$ and $t_{\mathrm{sp}}$ in our simulations that produce an increase in flux of $50 \%$ of the mid-transit depth (see Fig. 1, dashed line).

\subsection{Model calculations}

Given the assumption that a spot has either a circular or an elliptical shape with a total area $A_{\mathrm{sp}}$, our algorithm calculates analytically the light curve modulations due to stellar rotation using the relationship

$\frac{F_{\mathrm{sp}}}{F_{0}}=1-\frac{A_{\mathrm{sp}} \cdot \cos (\theta)}{\pi R_{\star}^{2}}\left(1-t_{\mathrm{sp}}^{4}\right) \cdot I\left(\theta, c_{1}, c_{2}\right)$,

where $\theta$ denotes the angle between the normal to the stellar surface and the line of sight towards the observer. The factor $I\left(\theta, c_{1}, c_{2}\right)$ accounts for the LD of the disk, which depends on the angle $\theta$ and, because we choose to use a quadratic LD parameterization, on the limb-darkening coefficients (LDC) $c_{1}$ and $c_{2}$.

When a planet transits in front of the star, our algorithm uses the model of Mandel \& Agol (2002) to calculate the transitinduced flux reduction. In Fig. 2 we show an example of some typical light curves that our algorithm produces. In this example the light curve of a Neptune-sized planet orbiting a K-type dwarf is shown; its noise level is adjusted to a star with a Kepler magnitude of $K_{\mathrm{p}}=13$ (black dots) along with the infinite signalto-noise case (red line).

The calculation of spot-crossing events involves intersections of circles and an ellipse, which we choose to perform numerically. To the left of the transit center there is an anomaly caused by a spot-crossing event. The overall light curve slope is caused by the flux variation due to the rotation of the star.

\subsection{General assumptions}

We briefly summarize the basic assumptions for the simulated light curves we use to study the effects of spot-crossing events on TTVs:

1. The planet crosses only one uniformly dark spot per transit.

2. The shape of the spot is either circular or elliptical.

3 . We consider relative spot temperatures $t_{\mathrm{sp}}$ of $0.3,0.5$, and 0.7 .

4. The fraction of the planet that covers the spotted area is chosen in such a way that the flux increment at mid-transit yields $50 \%(H=0.5)$, assuming an impact parameter $b=0$.

5. The noise level is adjusted to the precision of Kepler short cadence photometry for a $K_{\mathrm{p}}=13 \mathrm{mag}$ star (Gilliland et al. 2010).

6. We introduce the parameter signal-to-noise ratio of the transit (TSNR), which is the transit depth divided by the out-oftransit light curve noise level (see assumption 5).

7. Each data point represents an integration over $60 \mathrm{~s}$.

8. The rotation period of the star is fixed to 10 days.

\section{Simulation strategy}

Spot-crossing events cause deformations of the transit shape which, potentially, lead to a shift of the measured mid-transit time. We set up individual light curve simulations to address three questions:

1. How much are the measured mid-transit times affected by the longitudinal position of a spot during a spot-crossing event?

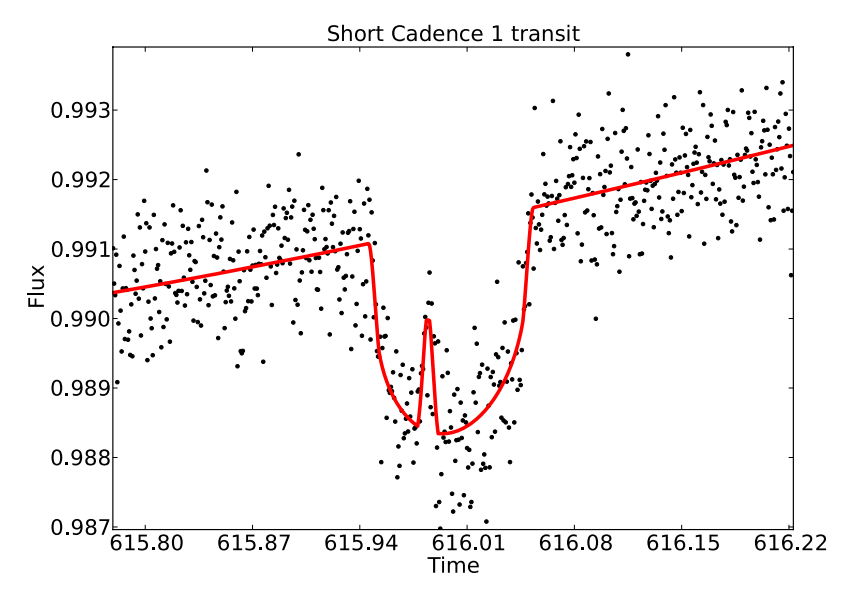

Fig. 2. Simulated transit of a Neptune-sized planet. The red line represents the infinite signal-to-noise data, while the noise of the black dots is similar to that of Kepler short cadence data. During the transit there is a spot-crossing event right after the ingress.

2. How do the orbital characteristics of the planet, i.e., its orbital period and impact parameter, influence the shift of midtransit times?

3. Does the influence of a spot-crossing event on timing measurements vary for different stellar types?

\subsection{Spot position}

The strength of the deformation of a transit profile caused by a spot-crossing event depends on the longitude of the spot. To study the effect on mid-transit times, we first consider a transit of a planet with an orbital period equal to the stellar rotation period. The planet has an impact parameter of $b=0$ and, thus, transits the center of the stellar disk. A spot with a relative temperature of $t_{\mathrm{sp}}=0.3$ is placed on the stellar equator at longitude $\lambda_{\mathrm{o}}$, which is the longitude of the spot at the epoch of the observation; $\lambda_{0}=0$ denotes the sub-observer point at the center of the disk, with $\lambda_{\mathrm{o}}=-90$ being the leading edge and $\lambda_{\mathrm{o}}=90$ the trailing edge of the disk.

We adopt a K-type dwarf host star $\left(T_{\text {eff }}=4300 \mathrm{~K}\right)$ with a quadratic LD law and use LDCs of $c_{1}=0.690$ and $c_{2}=0.034$ as provided by Claret et al. (2012) for the Kepler mission. We simulate light curves based on three different assumptions for TSNR: one for infinite TSNR, and two more with TSNR values equal to the expected precision for planets with $R_{\mathrm{pl}} / R_{\star}=0.05$ and $R_{\mathrm{pl}} / R_{\star}=0.1$ (see assumptions 5 and 6 in Sect. 2.4).

\subsection{Planetary orbit}

If the planet's orbital period $P_{\mathrm{pl}}$ is much smaller than the stellar rotation period $P_{\star}$, the spot's position hardly changes while the planet is crossing over it. If $P_{\mathrm{pl}} / P_{\star}$ increases, the spot starts to move significantly during its occultation by the planet. To estimate the strength of this effect on the measurement of mid-transit times, we study simulations with orbital periods of 5, 10, 20, 40, $80,150,300$, and 600 days. We also consider different impact parameters of $0 \leq b<1$ in combination with different spot longitudes $\lambda_{\mathrm{o}}$. The sizes of our test planets are $R_{\mathrm{pl}} / R_{\star}=0.1$ and $R_{\mathrm{pl}} / R_{\star}=0.05$. For this simulation the stellar inclination is fixed to zero and the host star has the same properties as described in Sect. 3.1. 


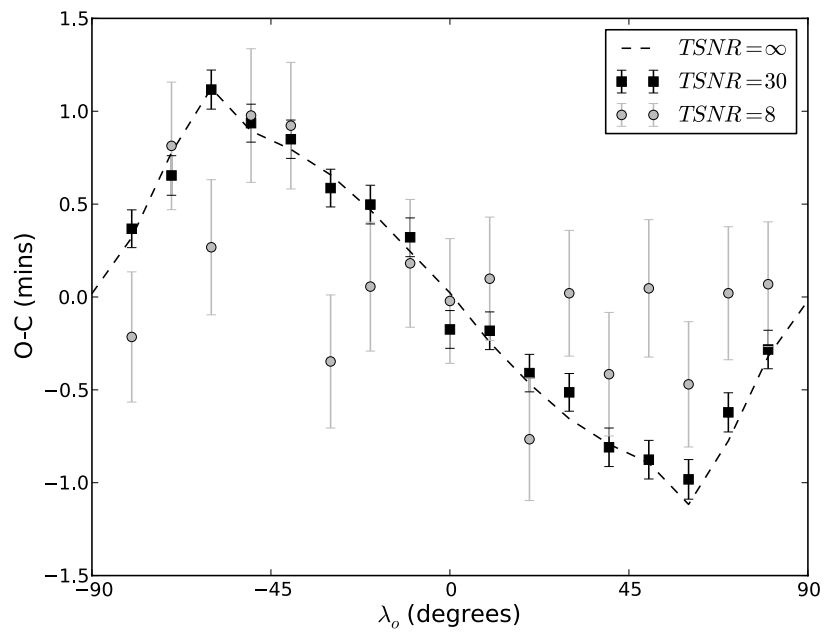

Fig. 3. $t_{\mathrm{sp}}=0.3$ simulation for different TSNR levels. The dashed line represents the case of infinite TSNR. The measurement error for transits with TSNR $=30$ is still small enough for the underlying modulation caused by the spots to be seen, whereas for TSNR $=8$ the spot signal vanishes in the noise.

\subsection{Stellar limb darkening and inclination}

Even though the effect of a spot-crossing event on TTV measurements should be dominated by the properties of the spot and the planet, we also investigate the influence of LDCs in detail. We consider main-sequence stars with effective temperatures of $3500 \mathrm{~K} \leq T_{\text {eff }} \leq 10000 \mathrm{~K}$ and use the corresponding LDCs of the Kepler band pass (Claret et al. 2012). Furthermore, we investigate how different stellar inclinations influence the measurement of mid-transit times. The planet in this setup has a size of $R_{\mathrm{pl}} / R_{\star}=0.1$ and an impact parameter of $b=0$. We vary the inclination of the star and the spot latitude.

\section{Results}

The light curves simulated in Sect. 3 generally contain a slope caused by the starspot located on the rotating surface (see Fig. 2). In order to remove this trend, we first identify the transit in the light curve, remove those parts of the light curve affected by the transit, fit a second-order polynomial to the remaining out-oftransit data, and divide the fit from out-of- and in-transit data to produce a normalized transit light curve. We then compute the mid-transit times and errors from these normalized transit light curves by using the model described by Mandel \& Agol (2002) combined with a Markov chain Monte Carlo (MCMC) sampler where the only free parameter is the mid-transit time.

\subsection{Spot position}

Here we present the results derived for the simulations described in Sect. 3.1. In Fig. 3 (dashed line) we show the difference $(\mathrm{O}-\mathrm{C})$ between mid-transit times determined from our simulated light curve and the input times for the different longitudes $\lambda_{\mathrm{o}}$ of the occulted spot.

Figure 3 (dashed line) also illustrates that the maximum deviation of TTV measurements caused by spot-crossing events is $\sim 1.2 \mathrm{~min}$ in this particular case. The maximum values are encountered when the spot is located between the center and the limb of the stellar disk at about $\lambda_{\mathrm{o}} \sim \pm 70^{\circ}$. An anomaly at the transit center has no influence on the derived mid-transit times. The spot-induced deviations also decrease towards the limb of

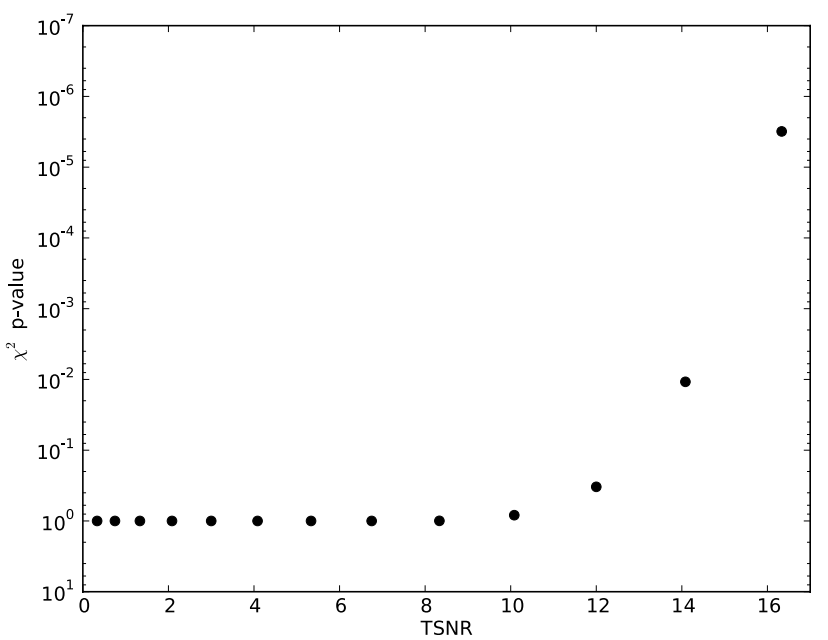

Fig. 4. p-values of the null hypothesis $\chi^{2}$ analysis for mid-transit times of transits with different TSNR values. The transits with TSNR $<15$ do not show statistically significant evidence of variations. As a result, the mid-transit times of better resolved light curves are more vulnerable to deviations caused by spot crossing events.

the star because the projected size of the spot decreases and LD reduces the flux contributing from these parts of the stellar disk.

The spot-crossing anomalies introduce positive variations when they occur before the mid-transit time and negative ones in the opposite case. When the planet occults a spot during ingress or egress, the measured transit duration is affected mostly because there is a pseudo-shift of the first or fourth contact time; the transit seems to start later or end earlier, respectively. Thus, there is a shift of the mid-transit time into the opposite direction of the spot-crossing event.

So far we have dealt with the case of infinite TSNR. Naturally, measured mid-transit times have errors that we determine using a MCMC algorithm. Measurements and their errors for the case of a planet with $R_{\mathrm{pl}} / R_{\star}=0.1$ are shown in Fig. 3, which corresponds to an TSNR of 30 and $R_{\mathrm{pl}} / R_{\star}=0.05$ equivalent to TSNR $=8$. For the larger planet the spot-induced deviations of the mid-transit times from the input is still detectable within the relatively small errors and follows the same pattern as for the infinite TSNR case. However, the errors for the smaller planet are much higher and a systematic modulation caused by spots is no longer clearly visible.

Assuming as a null hypothesis that no TTVs are produced by the spot, we perform a $\chi^{2}$ analysis on the TTVs (see Fig. 3) of transits with different TSNR. As shown in Fig. 4, the deviations caused by spot-crossing events start to disappear in the measurement noise for transits with TSNR $\lesssim 15$.

\subsection{Planetary orbit}

Based on the simulations described in Sect. 3.2, Fig. 5 presents the maximum deviation of mid-transit time measurements caused by spot-crossing events for different values of $P_{\mathrm{pl}} / P_{\star}$. The TTV maximum increases linearly with the transit duration, which in turn increases with the period ratio. For the same spotcrossing event a longer transit duration leads to a larger shift (e.g., Sanchis-Ojeda et al. 2011).

We also consider the possibility that spots might not always be circular. In Fig. 5 we also show our results for elliptical spots, which indicate that circular and elliptical spots with equal filling 


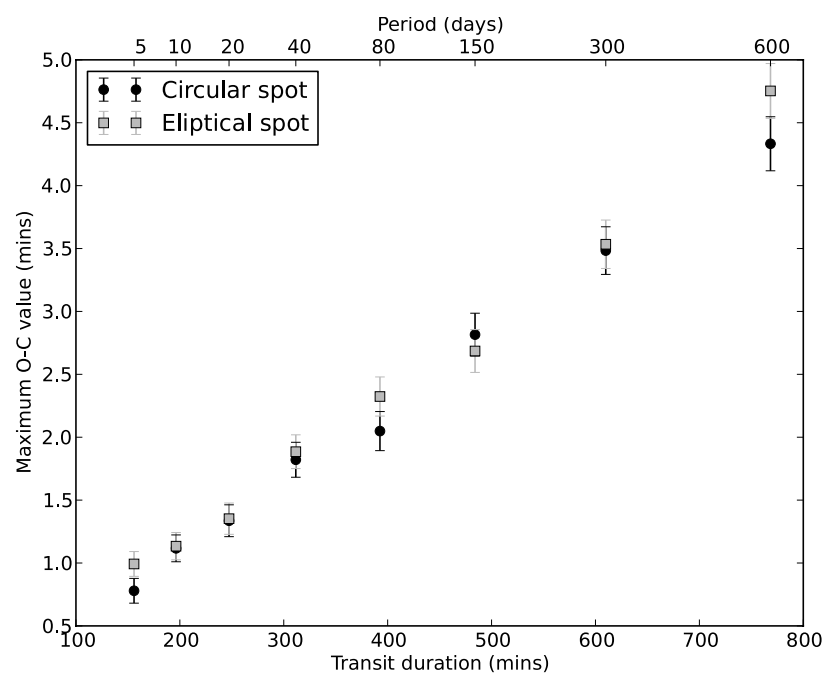

Fig. 5. Maximum deviation in the mid-transit time of a planet with TSNR $=30$ for different transit durations. As the period and hence the transit duration increase, the maximum deviation grows larger. The black dots refer to the maximum values caused by circular spots; the gray squares refer to elliptical spots.

factors lead to virtually identical results. We conclude that the precise spot geometry appears to be of minor importance.

Next we consider how the derived transit times depend on the impact parameter of the transits. Figure 6 shows how the difference between measured and calculated mid-transit times changes with the impact parameter of the planet. Owing to the LD of the stellar disk and to geometrical effects, the transit profile becomes more V-shaped for larger impact parameters, and the maximum of the residuals moves closer to the center of the star. The change of the transit shape increases the influence of the spot-crossing event on the measurement of the mid-transit time. On the other hand, for high values of the impact parameter, the amplitude of the $\mathrm{O}-\mathrm{C}$ becomes smaller due to the shorter transit duration (see Fig. 5) . Therefore, the additional deviation that the deformation of the transit introduces is canceled by the reduction of the transit duration. As a result, there is no significant difference in the amplitude of the residuals for different impact parameters.

\subsection{Stellar limb darkening and inclination}

In order to study how LD affects the timing deviations we used a planet size of $R_{\mathrm{pl}} / R_{\star}=0.1$ and a relative spot temperature of $t_{\mathrm{sp}}=0.3$, assuming a period ratio equal to unity. The strength of the measured deviations does not significantly depend on the LDCs. However, for lower effective temperatures the region of large deviations introduced to the mid-transit time is larger and its inner boundary moves closer to the center of the star.

To better illustrate the influence of LD on the effect of transit time measurements, we simulated light curves with different linear and quadratic LDCs in the range of zero to unity. The results are presented in Fig. 7, where the left panel shows the results for different linear LDCs with $c_{2}=0$ and the right panel for different quadratic LDCs with $c_{1}=0$. While the spot-crossing anomaly moves from one side of the transit to the other, the measured shift changes in a similar way to what has been shown in Fig. 3. However, Fig. 7 illustrates that the influence of LD on the shape of the curve is rather large. For small LDC values there is only a spot-induced shift of the transit time at ingress and egress and

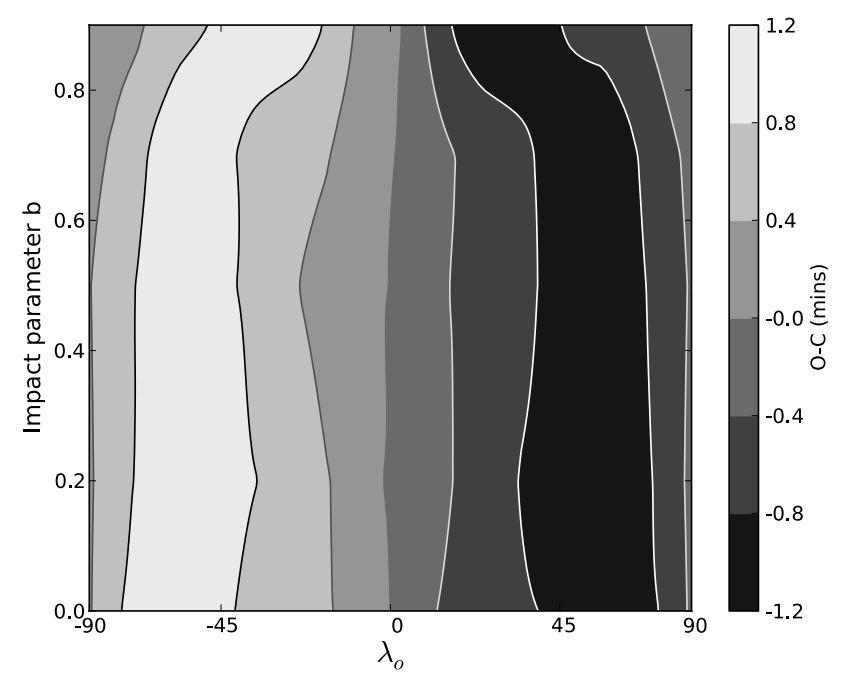

Fig. 6. TTVs for a simulation of a planet with TSNR $=30$ (contour map) as a function of the longitude $\lambda_{\mathrm{o}}$ and the impact parameter $b$. As shown in Fig. 3, O-C has positive values for spot-crossing events before $\lambda_{\mathrm{o}}=0$ and negative values otherwise. While there is almost no difference in the maximum values, their positions shift to longitudes closer to the center of the star for higher impact parameters.

hardly a shift in between because, in the absence of LD, the transit has a box-shaped profile and is flat at the bottom. The model does not have the flexibility to compensate an anomaly caused by the spot in the flat part of the transit. Thus, this anomaly cannot be fitted and the best fit results ignore the spot.

However, this changes for larger LDCs because the transit shape becomes rounder and the bottom is no longer flat. The stronger the LD, the higher the curvature, and a shift of the model can lead to a better fit even if the spot signature is closer to the center. This effect is stronger for the linear component because it influences the center of the transit much more than the quadratic LDC does. The latter has its largest influence at the ingress and egress which is the reason why in this case the curves show greater change in the outer parts and only a little at the center. Although the influence of LD appears to be large (see Fig. 7), for the relevant temperatures considered in our simulations the LDCs change very little and stay at roughly $c_{1}=0.7$ and $c_{2}=0.1$. For small changes in the LDCs, the curves in Fig. 7 change only slightly.

Finally, we also investigated how different values for the inclination of the rotation axis of the star affect the measured transit times. However, we could not detect a dependence in the maximum amplitude of the spot-induced TTVs on the stellar inclination.

\section{Sequence of consecutive transits}

So far we have studied only the effects of a spot-crossing event on the TTV measurements of a single transit for different parameter combinations. However, we have not yet investigated series of consecutive transits of the same system. According to our previous results, a spot-crossing event can cause deviations of the mid-transit times of up to a few minutes. The amplitude alone, however, does not tell us whether it is possible to produce a spurious signal in the timing measurements from a sequence of transits that could mimic the signal of a true physical companion in the system. In this case the activity would lead to a false positive detection of a body that does not actually exist. 

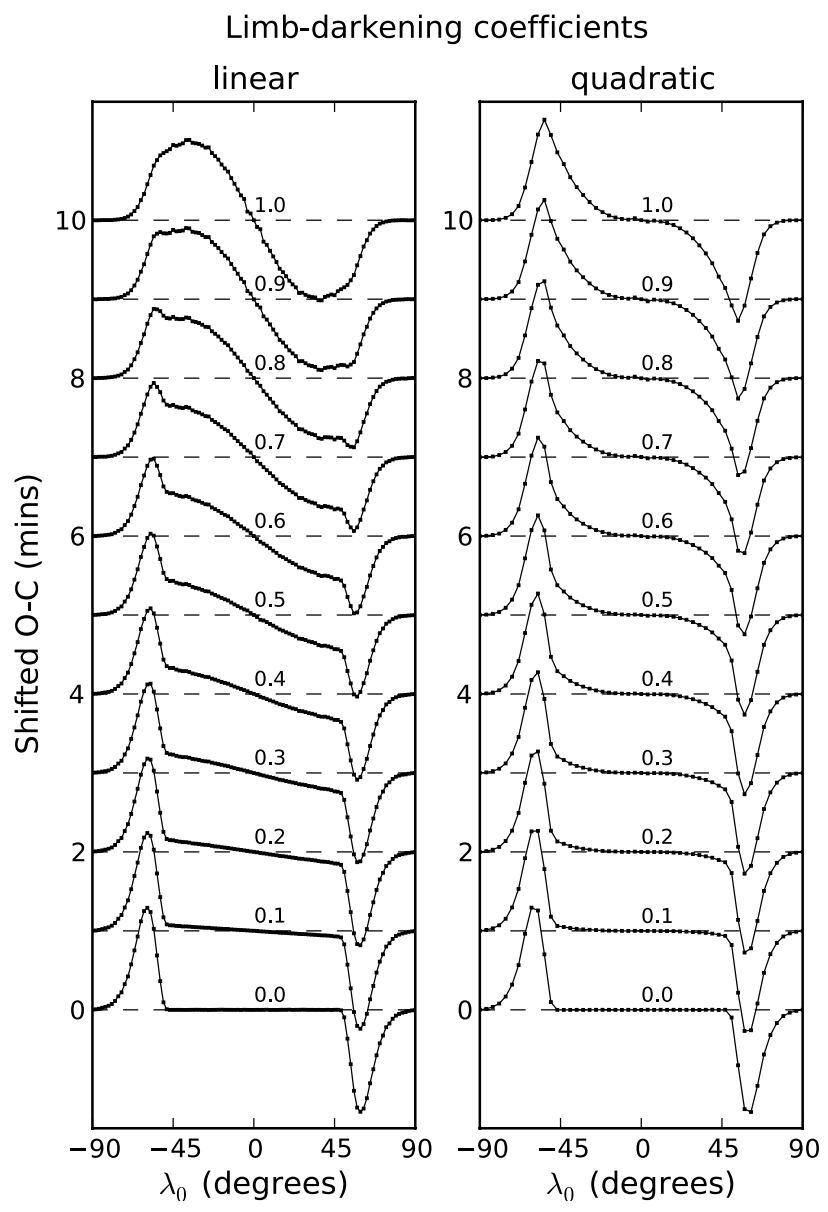

Fig. 7. Effect of different LDCs on the measured mid-transit time for transits with infinite TSNR and spot-crossing events in various longitudes $\lambda_{\mathrm{o}}$. The panels refer to linear (left) and quadratic (right) LDCs with values between unity (top) and zero (bottom).

It is rather difficult to study such problems in simulations because the results always sensitively depend on the selected input parameters. If we choose some specific spot configuration with spot lifetimes and differential rotation, etc., our results would strongly depend on these assumptions. We try to minimize this by choosing a simple, straightforward approach which considers a worst-case scenario: we assume that for all transits in a light curve the spot is always occulted if it is located on the visible hemisphere and that the spot has an infinite lifetime. This is certainly not what one expects to actually find; spots are not always located exactly under the planet path and they certainly have limited lifetimes. Furthermore, we adopt a comparatively large spot. However, in this way our results will give an estimate of what might be observed in the most problematic cases.

\subsection{Simulation setup}

We set up 7500 simulations in such way that for each individual simulation a series of 100 consecutive transit light curves of the same system are calculated. We consider different TSNR values and orbital periods of the planet (measured in days), which are both randomly drawn from a uniform distribution between 1 and 30. The stellar rotation period is always fixed to 10 days. We assume only one spot on the surface which is always occulted when located on the visible hemisphere. If the planet crosses an unspotted disk, its transit will not be deformed by any spot-crossing feature; nonetheless, the transit will continue to be part of the sequence. The spot lifetime is assumed to be infinite and its covered area is set to follow the assumption given in item 4 in Sect. 2.4

We then measure the mid-transit times of the simulated transits as described in Sect. 4 (including their measurement uncertainties using MCMC) and calculate the residuals by subtracting the expected values $(\mathrm{O}-\mathrm{C})$. Additionally, we calculate the mean of the residuals and subtract it. The Generalized LombScargle periodogram (Zechmeister \& Kürster 2009) is then used to determine the period $P_{\max }$ in the TTVs with the smallest false alarm probability (FAP) for each individual simulation. Finally, for each simulation we calculate the amplitude of the TTVs using the relationship

$A_{\max }=\left[(\mathrm{O}-\mathrm{C})_{\max }-(\mathrm{O}-\mathrm{C})_{\min }\right] / 2$.

\subsection{Simulation results}

In Fig. 8 we show the FAP of the most significant period as a function of the TSNR for each of our simulations. The average FAP decreases with increasing TSNR. This reflects the fact that transit times calculated from light curves with small TSNRs have relatively large uncertainties which leads to higher FAPs when searching for periods. The majority of periods for transits with TSNRs $\gtrsim 15$ have FAPs $\lesssim 10^{-3}$, which we considered significant enough not to be spurious signals. Although in our simulations these periods are caused by spots, a reliable distinction of whether such a period is caused by spots or additional bodies in the system might be quite complicated in real observations.

Figure 9 shows $A_{\max }$ as a function of the period ratio for all individual simulations. We divided the results into two groups, amplitudes coming from simulations with a FAP $<10^{-3}$ (red points) and amplitudes with larger FAPs (black points). For the latter group the uncertainties of the transit time measurements are high (TSNR is usually small) and the amplitude primarily reflects the scatter of the TTVs due to noise. For the first group the systematic variation due to spots is larger than the uncertainties (high TSNR) and the amplitude represents the effect the spots have on the measurement of the TTVs. The FAP $<10^{-3}$ points show much smaller scattering and are limited to a region close to the lower edge of the measured amplitudes. As expected, the majority of these points lie under the maximum deviation values (dashed line), which we present in Fig. 9. This region is basically defined by the planetary period and therefore the transit duration, because the maximum amplitude of a spot-crossing event becomes smaller for smaller transit durations.

An interesting feature of Fig. 9 is the localized minima of amplitudes at integer period ratios. At these resonance periods the spot-crossing feature hardly changes position in the transit profile and the spot-induced timing variation are small. A period ratio of exactly one (two, three, etc.) means the TTVs are all on one level and vary only because of noise.

In Fig. 10 the period with the highest FAP determined from the $\mathrm{O}-\mathrm{C}$ diagram of each individual simulation is plotted over the period ratio. The lower envelope is determined by the Nyquist frequency. The highest periods that can be determined are roughly defined by the time span covered by the TTVs. The up-and-down patterns shown in Fig. 10 result from the different TSNRs of the simulations. For low FAPs (red points) maxima at resonances of period ratios $(0.5,1,1.5$, etc.) and minima in between can be seen. In a system where the periods of star and planet are not integral multiples, the position of the spotcrossing event changes from one transit to the next. As the period 


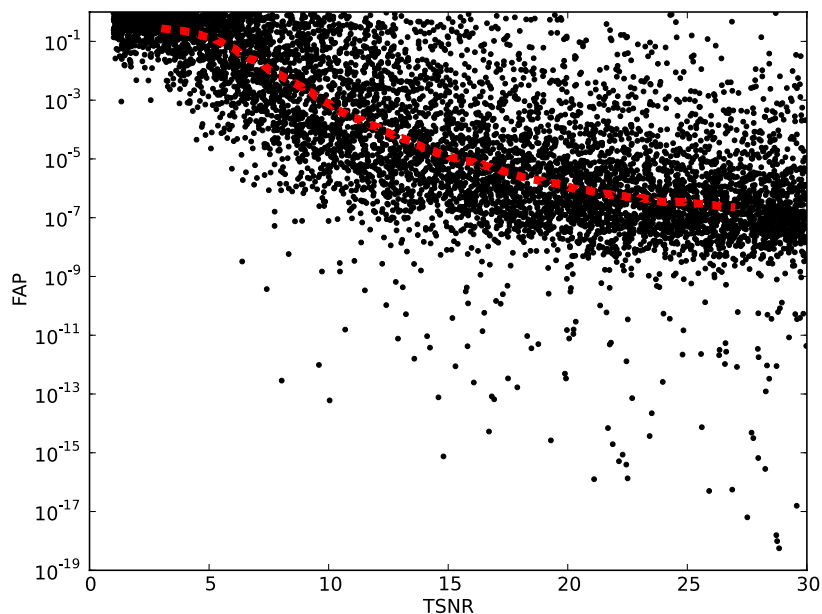

Fig. 8. FAP of the highest peak in the Generalized Lomb-Scargle periodogram over the TSNR of the planetary transits for all 7500 simulation. On average, the strongest periods in the simulations become more significant for higher TSNRs. The red line demonstrates the running median of the FAP in TSNR bins of six. Most of the points larger than TSNR $\gtrsim 15$ have FAPs below $10^{-3}$.

ratio $P_{\mathrm{pl}} / P_{\star}$ moves closer to a resonance, the difference between the longitudes $\lambda_{\mathrm{o}}$ of the spot-crossing events in two consecutive transits becomes smaller. This leads to a lower frequency TTV, which is interpreted as a higher TTV period by the periodogram. Exactly at the resonance the TTVs are flat and the periods go down to the Nyquist frequency. This is also true for period ratios at $0.5,1.5$, and so on; however, not every transit shows a deformation caused by a spot. At ratio 0.5 , for example, the spot is seen only every second transit, which results in one level of transits that are not disturbed by the spot and a second level of transits which are all shifted by the same value. For very low noise the amplitude is then basically given by the difference between the two levels.

Going to larger FAPs, we see a second pattern (made up of red and black points in Fig. 10), which is reminiscent of the previous structure but peaks only at integer resonances and goes down to the Nyquist frequency at $0.5,1.5$, and so on. This happens when the uncertainties of the individual mid-transit time measurements are about equal to the amplitude caused by the spot-crossing effect. For simulations with even higher FAPs, we end up with the points that are randomly distributed because the uncertainties of the mid-transit times are larger than potential spot-induced variations.

\subsection{Can spots mimic bodies in transit sequences?}

We emphasize again that our simulations show a worst-case scenario. In the majority of planetary systems, periodic signals in TTVs caused by spots should be much weaker and less coherent. In reality, the spots grow and decay, change their positions, and are probably smaller than we assume in our simulations. Such changes will always lead away from the clear patterns shown in Fig. 10 (red points) to a random distribution of points.

However, we argue that it is possible to observe a periodic signal mimicking TTVs of a potential companion in the planetary system caused by spot-crossing events. For high TSNRs, period ratios close to resonances are particularly dangerous. A careful inspection of the transit shapes and their deformations should provide additional information on whether the determined mid-transit times are reliable or subject to dispute. In

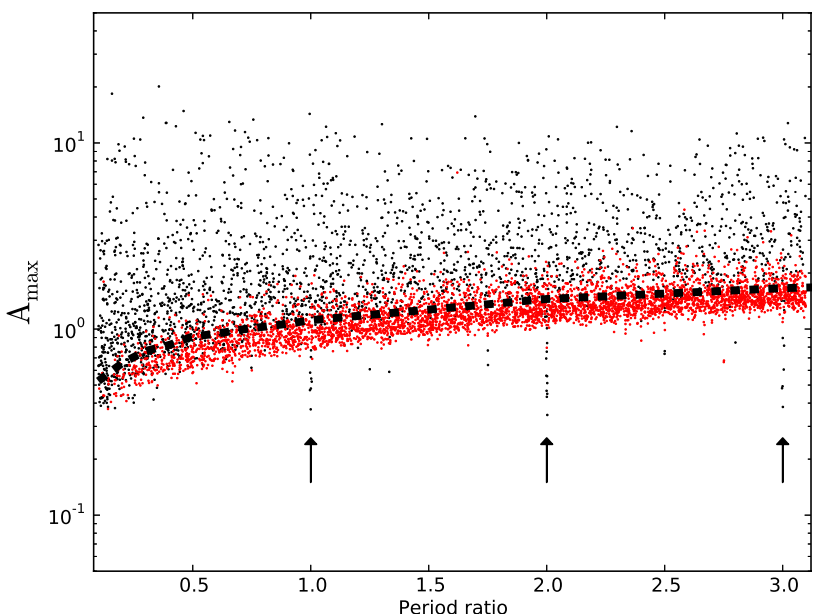

Fig. 9. Amplitude of the TTV measurements over the period ratio for all individual simulations. The red points indicate all simulations for which the determined period has a FAP $<10^{-3}$, which are the simulations with high TSNRs (see Fig. 8). The black dashed line indicates the maximum amplitude values given by Fig. 5 . The arrows indicate the localized drops in amplitudes at integer period ratios (see Sect. 5.2).

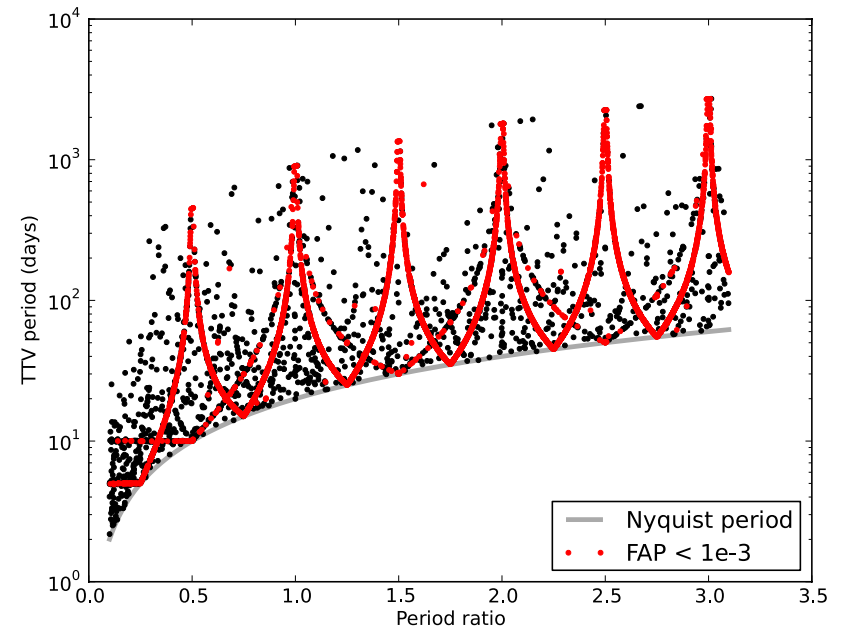

Fig. 10. For each individual simulaiton, the most significant period is determined from the TTVs over the input period ratio. The gray line indicates the lower limit of possible periods defined by the Nyquist frequency. The red points indicate periods with $\mathrm{FAP}<10^{-3}$.

many cases the TTVs due to spots should show odd behavior such as the splitting of points into two or more levels or alternating intervals of strong and weak variability, which could be valuable indications that the TTVs are possibly due to spots. Unfortunately, depending on the exact properties of star, spot, and planet, TTVs do not necessarily have to show such odd behavior.

Whenever a significant periodic signal is found in noisy TTVs, it should be interpreted carefully especially if the star shows signs of activity. If small-scale features in the transit profiles are hard to discern and if the errors in the transit times are on the order of (or even larger than) the deviations due to potential spots, one could still end up with a significant period. Whether this period is caused by spots or a yet unknown body in the system will be hard to determine. 


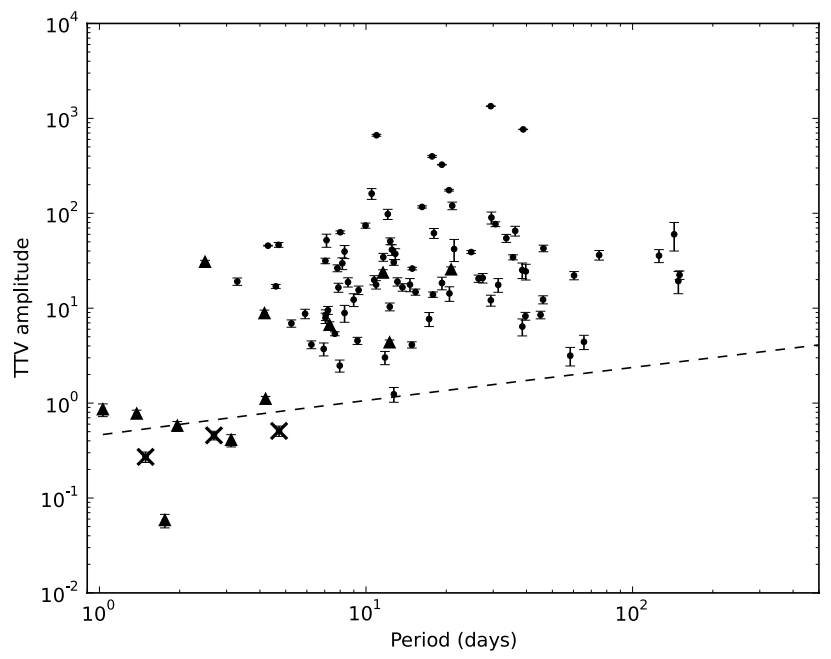

Fig. 11. TTV amplitudes reported by Mazeh et al. (2013). The dashed line denotes a linear fit to the maximum amplitudes for TSNR $=30$ (see Fig. 5). The points marked with $X$ are found to have periods related to the stellar rotation; all of them lie well below our predicted amplitude maximum caused by spots. The points marked with triangles are taken as spurious which could have been caused by other sources capable of inducing TTVs (i.e., sampling or eclipsing binaries).

\section{Comparison to observations}

In Fig. 11 we show the cases of TTV detections as presented by Mazeh et al. (2013). In their discussion regarding those TTVs detections Mazeh et al. (2013) suggest that three of them show a correlation between the period of the TTVs and the rotational period of the star that was determined from its light curve. The TTV amplitudes for these planets lie well below the upper limit we calculated from our simulations, which supports our result that larger amplitudes caused by spots are unlikely. Larger TTV amplitudes might also be modified by starspots but are hardly exclusively created by them.

In a recent article Masuda et al. (2015) present the light curve of the KIC 6543674 triple star system, which consists of a shortperiod (2.4 days) inner binary with a companion in a wide eccentric orbit. Their study of the system presents eclipse timing variations (ETVs) with amplitudes of $\sim 4$ min which do not seem to be caused by any known companion. They imply that these ETVs can be explained by the existence of starspots; however, they do not model them. Although we do not study ETVs of stars in this paper, a spot-crossing event of a star should be comparable to our simulations with planets. Spot-eclipses should cause shifts from the expected mid-eclipse time due to the deformation of the eclipse shape as well.

The residual ETVs resemble quite closely the longitudedependent spot-crossing pattern we show in Fig. 3. In order to create such ETVs, the period ratio between the orbital period of the occulting body and the rotational period of the occulted star must be very close to, but not exactly equal to an integer value. Interestingly, the ETVs do not show flat regions with no modulation, which one would expect if no spot is on the visible hemisphere. Instead the pattern continues very regularly, which indicates the existence of two or more large active regions, non-uniformly distributed on the surface of the occulted star. Another interesting feature is the long lifetime of these active regions. The modulation in the ETVs evolves so slowly that a reminiscent pattern repeats for about three years before it significantly changes.
The amplitude of the measured ETVs appears to be larger than predicted by our simulations for exoplanetary transits with equal duration times; the eclipses of the inner KIC 6543674 system last about $360 \mathrm{~min}$, which according to Fig. 5 should lead to maximum amplitudes of about $2 \mathrm{~min}$. To check if we could reach the amplitude of the Masuda et al. (2015) ETVs, we run a simulation setup with the properties of this system and obtain ETVs with comparable amplitudes in quite a few cases. This result also agrees with the amplitudes of spot-induced ETVs from other binaries shown by Orosz (2015). There are two reasons for the deviation between the maximum amplitudes of spot-induced TTVs and ETVs, the shape of the eclipse and the area of spots covered by the occulting body. The eclipses of binary stars with comparable sizes usually show only the ingress and the egress and not the flat part in between. Therefore, spot-crossing anomalies can cause significant TTVs for spots at all possible longitudinal positions. Owing to the large size of the occulting body, it is also likely that large areas of spots on the occulted body are eclipsed, which also leads to stronger ETVs.

A detailed modeling of this system, including spots, is needed to evaluate whether these two arguments are sufficient to explain the observed ETVs - or even if spots are an appropriate explanation for these ETVs at all. Although this is beyond the scope of this work on exoplanets, we emphasize that if these ETVs are really due to starspots they resemble the results of our simulations quite nicely and demonstrate that clear repetitive modulations due to spots over timescales of years are possible, and the probability of misinterpreting them as signals of an unknown body is not negligible.

\section{Discussion}

Our simulations of spot-induced transit timing variations suggest that even large spot-crossing anomalies in transit profiles should not lead to TTV amplitudes larger than a few minutes (Figs. 5 and 9). The amplitude depends very strongly on the transit duration and the position of the spot-crossing event. As shown in Fig. 3, the uncertainties of the measured mid-transit times primarily depend on the TSNR and transit duration, but only little on the characteristics of the spot-crossing events. Specifically, the uncertainties do not depend on the position of the spot at the time of the transit, whereas the TTV amplitude clearly does. Thus the uncertainties should not be used as a diagnostic for spot-induced TTVs, despite the deformation of the transit light curve, even for the rather large spots assuming in our simulations.

The amplitude of the spot induced TTVs is independent from the inclination of the star, as we discuss in Sect. 4.3. Nevertheless, in the case of low stellar inclination it is possible that a spot-crossing event can occur at the cross section of the planetary orbit and the active latitudes of the star. This has an impact on the results given in Sect. 2; due to the fixed position of the spot-crossing events, the values of the TTVs amplitudes are going to be distinct and not continuous. Such O-C diagrams produce periodicities that have very little significance which have a low probability of being considered real.

The maximum mid-transit time deviations derived from our simulations are significantly smaller than the maximum O-C values calculated from Mazeh et al. (2015) and Oshagh et al. (2013). The reason for this discrepancy is our assumption that the in-transit flux increment due to the spot occultation is not larger than $50 \%$ of the mid-transit depth. In our opinion this value is a better representation of observational evidence, since 
we do not know of a single case where the induced anomaly is $\gtrsim 50 \%$ of the transit depth.

\section{Conclusions}

Our simulations were geared to study TTVs induced by starspots using high-precision photometric data without any red noise sources. While it is relatively safe to apply our results to data obtained by space missions, it is difficult to estimate the behavior of such spot-induced TTVs on light curves with significant contribution of red noise (i.e., scintillation).

Finally, we summarize the most important results of our simulations on spot-induced TTVs of exoplanets:

- In agreement with the results of Oshagh et al. (2013) and Barros et al. (2013), spot-crossing events can cause shifts of the measured mid-transit times. These shifts can cause a specific pattern depending on the longitude $\lambda_{\mathrm{o}}$ of the spot during the crossing event (see Fig. 3).

- The amplitude of the shift is determined by the temperature of the spot relative to the photospheric temperature, the fraction of the planet covering spots, and the duration of the transit.

- The shape of the spot or the stellar inclination during a spotcrossing event do not seem to significantly affect the midtransit times.

- We find only a weak dependence of the measurement uncertainties on longitude $\lambda_{0}$. We conclude that they are no reliable indicators for how much individual timing measurements are affected by spots.

- The impact parameter of the planet and the limb darkening of the star influence the shape of the transit profile, which affects the TTV measurements. However, this seems to be a minor contribution compared to the other influences.

- A sequence of transits affected significantly by spots can create a statistically significant periodical signal. In special cases the spot-induced TTVs could even show a clear, persistent modulation imitating the existence of a sub-stellar companion. However, these cases should be rare and a careful analysis of the data should indicate a potential influence of starspots, especially for systems with high TSNR.

- Extra attention should be paid to TTVs with amplitudes $\lesssim 1 \%$ of the transit duration (see Figs. 5, 9, and 11).

- The detection of significant spot-induced periods in TTVs depends on the TSNR, and according to our simulations is unlikely for TSNR $\lesssim 15$. A detection of periods for TSNR $>15$ should be treated cautiously, especially if they are attributed to unknown bodies in the system.

Acknowledgements. P.I. acknowledges funding through the DFG grant RTG 1351/2 "Extrasolar planets and their host stars". K.F.H. is supported by DFG grant HU 2177/1-1.

\section{References}

Agol, E., Steffen, J., Sari, R., \& Clarkson, W. 2005, MNRAS, 359, 567

Baglin, A., Auvergne, M., Barge, P., et al. 2006, in ESA SP 1306, eds. M. Fridlund, A. Baglin, J. Lochard, \& L. Conroy, 33

Barros, S. C. C., Boué, G., Gibson, N. P., et al. 2013, MNRAS, 430, 3032

Basri, G., Walkowicz, L. M., \& Reiners, A. 2013, ApJ, 769, 37

Claret, A., Hauschildt, P. H., \& Witte, S. 2012, VizieR Online Data Catalog: $\mathrm{J} / \mathrm{A}+\mathrm{A} / 546 / \mathrm{A} 14$

Czesla, S., Huber, K. F., Wolter, U., Schröter, S., \& Schmitt, J. H. M. M. 2009, A\&A, 505, 1277

Fabrycky, D. C., Ford, E. B., Steffen, J. H., et al. 2012, ApJ, 750, 114

Gilliland, R. L., Jenkins, J. M., Borucki, W. J., et al. 2010, ApJ, 713, L160

Huber, K. F., Czesla, S., Wolter, U., \& Schmitt, J. H. M. M. 2009, A\&A, 508, 901

Koch, D. G., Borucki, W. J., Basri, G., et al. 2010, ApJ, 713, L79

Mandel, K., \& Agol, E. 2002, ApJ, 580, L171

Masuda, K., Uehara, S., \& Kawahara, H. 2015, ApJ, 806, L37

Mazeh, T., Nachmani, G., Holczer, T., et al. 2013, ApJS, 208, 16

Mazeh, T., Holczer, T., \& Shporer, A. 2015, ApJ, 800, 142

Nesvorný, D., Kipping, D., Terrell, D., et al. 2013, ApJ, 777, 3

Orosz, J. A. 2015, ASP Conf. Ser., 496, 55

Oshagh, M., Santos, N. C., Boisse, I., et al. 2013, A\&A, 556, A19

Rabus, M., Alonso, R., Belmonte, J. A., et al. 2009, A\&A, 494, 391

Sanchis-Ojeda, R., Winn, J. N., Holman, M. J., et al. 2011, ApJ, 733, 127

Szabó, R., Szabó, G. M., Dálya, G., et al. 2013, A\&A, 553, A17

Wolter, U., Schmitt, J. H. M. M., Huber, K. F., et al. 2009, A\&A, 504, 561

Zechmeister, M., \& Kürster, M. 2009, A\&A, 496, 577 